\title{
FITOTECNIA
}

\section{MANEJO INTEGRADO DE PLANTAS DANINHAS NA CULTURA DO MILHO $\left(^{1}\right)$}

\author{
ANDERSON LUIS NUNES $\left({ }^{2}\right)$; MICHELANGELO MUZELL TREZZI $\left({ }^{3 *}\right)$; CLENIO DEBASTIANI $\left({ }^{4}\right)$
}

\begin{abstract}
RESUMO
Estratégia de manejo de plantas daninhas pode ser realizada de forma integrada com o método químico na redução da utilização de herbicidas na cultura do milho. O objetivo deste trabalho é avaliar o efeito do manejo integrado do espaçamento entre linhas, ou de híbridos de milho com características morfológicas distintas e redução dos níveis de herbicidas, para controlar plantas daninhas na cultura do milho. O experimento foi instalado em delineamento de blocos ao acaso, com quatro repetições, arranjado em um fatorial $2 \times 2 \times 5$, em que: o primeiro fator foram os híbridos Penta (superprecoce, com folhas mais planas) e Flash (superprecoce com folhas mais eretas); o segundo fator foram os espaçamentos entre linhas de milho $(0,45$ e $0,9 \mathrm{~m})$ e o terceiro fator os níveis de manejo de plantas daninhas (capinado, sem capina e atrazine + foramsulfurom + iodosulfuron nas doses de 3000+45+3, $2250+33,8+2,3$ e $1500+22,5+1,5 \mathrm{~g} \mathrm{ha}^{-1}$ i.a.). Foram avaliados a altura e o ângulo foliar vertical das plantas, o número de plantas daninhas e a matéria seca de plantas daninhas por área. O ângulo foliar de plantas de milho torna-se mais planófilo com a adoção de espaçamentos reduzidos entre linhas, mas outras características de plantas de milho estão envolvidas no desempenho dos espaçamentos. O uso do espaçamento de $0,45 \mathrm{~m}$ entre linhas não contribui para a redução dos níveis de herbicidas para a cultura, mas sua adoção beneficia o manejo de plantas daninhas e o rendimento de grãos do híbrido Flash, embora não seja vantajosa para o híbrido Penta.
\end{abstract}

Palavras-chave: Zea mays, arranjo populacional, controle químico.

\section{ABSTRACT \\ INTEGRATED WEED MANAGEMENT IN MAIZE CULTIVATION}

Strategies to weed control in maize cultivation can be combined with chemical control to provide reduction of herbicide use. The aim of this work was to evaluate integrated management of plant row reduction, use of corn hybrids with contrasting morphological characteristics and reduction of herbicide levels to weed control. The experiment was carried out in a completely randomized block design with four replications in a $2 \times 2 \times 5$ factorial. The first factor was maize hybrids such as Penta (super-early season maize with flatter vertical leaf angle) and Flash (super-early season maize and more upright vertical leaf angle). The second factor was maize row width ( 0.45 and $0.90 \mathrm{~m}$ ) and the third factor was the weed management system (hoeing, no hoeing, and atrazine + foramsulfurom + iodosulfuron at $3000+45+3,2250+33.8+2.3$ and $1500+22.5+1.5 \mathrm{~g}$ a.i. $\mathrm{ha}^{-1}$ ). It was evalued the plant height and vertical leaf of corn plants, the number and dry matter of weeds per area. The vertical leaf angle of corn plants became flatter with the adoption of reduced row spacing, but other characteristics of corn plants were involved in the performance of spacing. The use of the narrow row spacing did not contribute to reducing levels of herbicides, but narrow row spacing benefits ony the weed management and grain yield of hybrid Flash.

Key words: Zea mays, population arrangement, chemical control.

( $\left.{ }^{1}\right)$ Recebido para publicação em 18 de março de 2008 e aceito em 23 de outubro de 2009.

$\left({ }^{2}\right)$ Curso de Agronomia da Universidade Tecnológica Federal do Paraná (UTFPR), Campus Pato Branco, Via do Conhecimento, km 01, 85503-390 Pato Branco (PR).E-mail: nunes.ander@gmail.com.

$\left({ }^{3}\right)$ Curso de Agronomia da Universidade Tecnológica Federal do Paraná (UTFPR), Campus Pato Branco, Via do Conhecimento, km 01, 85503-390 Pato Branco (PR).E-mail: trezzi@utfpr.edu.br $\left(^{*}\right)$ Autor correspondente.

$\left({ }^{4}\right)$ Responsável técnico, Sementes Lavoura S\&A, Rua Guarani, 760, 85501-050 Pato Branco (PR). E-mail: clenio@lavourasa.com.br. 


\section{INTRODUÇÃO}

O manejo integrado de plantas daninhas (MIPD) é considerado a principal ferramenta para reduzir o impacto ambiental dos herbicidas. O MIPD baseia-se na integração de métodos de controle, tornando os sistemas de cultivo desfavoráveis às plantas daninhas, minimizando seus efeitos. As estratégias podem ser utilizadas em conjunto com o método químico, permitindo a redução da utilização de herbicidas.

A interceptação de radiação pela cultura do milho pode ser aumentada pelo uso de espaçamentos mais estreitos entre linhas (KUnZ et al., 2007; BEGNA et al., 2001) e densidades mais elevadas (BEGNA et al., 2001). Além de modificar quantitativamente a disponibilidade de radiação para porções inferiores do dossel, alterações no arranjo de plantas podem modificar a qualidade da radiação que chega até as plantas. A proximidade entre plantas diminui a razão vermelho:vermelho extremo (R:FR), resultando em modificações morfofisiológicas nas plantas (BALLARÉ et al., 1990; SMITH, 2000; MADDONNI et al., 2002). Dentre as modificações, estão a angulação foliar planófila, o aumento da estatura das plantas, o aumento do comprimento, a redução da largura foliar (MADDONNI et al., 2001) e o aumento do número de folhas perpendiculares às linhas de cultivo (MADDONNI et al., 2001; RAJCAN et al., 2004).

A adoção de espaçamentos reduzidos entre linhas, em relação aos convencionalmente utilizados $(0,8$ a $0,9 \mathrm{~m})$, pode, simultaneamente, aumentar o rendimento de grãos da cultura do milho (Argenta et al., 2001a; SHRESTHA et al., 2001; JoHnSON e HoverSTAD, 2002; Strieder, 2006) e suprimir o crescimento de plantas daninhas, devido à maior quantidade de radiação interceptada pelo dossel da cultura, reduzindo a dependência de herbicidas (TEASDAle, 1995). No entanto, Johnson e Hoverstad (2002) observaram que a redução dos espaçamentos entre linhas de milho não reduziu a infestação nem o crescimento de espécies precoces ou tardias de plantas daninhas e também não influenciou o momento da aplicação de herbicidas.

Híbridos com arquitetura moderna, ou seja, com menor estatura, folhas mais eretas e em menor número possibilitam maior penetração de radiação para a porção inferior do dossel, reduzindo a competição intraespecífica, mesmo com utilização de densidades mais elevadas (Almeida et al., 2000; Argenta et al., 2001b). O uso desses híbridos também aumenta a razão R:FR e reduz as taxas de esterilidade da inflorescência feminina (SANGOI et al., 2003), componentes importantes para atingir maiores produtividades de milho. No entanto, alterações na quantidade e qualidade da radiação solar proporcionadas pelos híbridos mais modernos, podem representar maior emergência e desenvolvimento de plantas daninhas junto à cultura.

A redução de doses de herbicidas não é uma prática que possa ser utilizada indiscriminadamente em culturas, pois as doses de herbicidas são recomendadas em quantidades que asseguram amplo espectro de ação, sob diversos níveis de suscetibilidade e condições ambientais, na maioria das vezes, diferentes daquelas consideradas ideais para a ótima ação do herbicida (VIDAL, 2002).

As hipóteses desse trabalho foram: a) que características morfológicas de híbridos de milho, como ângulo foliar e estatura, influenciam a interceptação de radiação solar e podem modificar a infestação de plantas daninhas na cultura; b) a cobertura mais rápida do solo pela cultura e a menor passagem de radiação para o interior do dossel, resultantes da redução do espaçamento entre linhas de cultivo, podem inibir o desenvolvimento das plantas daninhas e assim possibilitar a redução de doses de herbicidas para seu controle.

Objetivou-se, com este trabalho, avaliar o efeito do uso integrado de híbridos com características morfológicas distintas, reduções do espaçamento entre linhas e doses de herbicidas, para controlar plantas daninhas na cultura do milho.

\section{MATERIAL E MÉTODOS}

O experimento foi desenvolvido em Latossolo Vermelho Distrófico no município de Pato Branco. O clima da região é classificado como $\mathrm{Cfb}$, segundo Köppen.

A semeadura da cultura ocorreu em 17 de outubro de 2003, sob delineamento de blocos ao acaso com quatro repetições, com arranjo fatorial $2 \times 2 \times 5$, em que o primeiro fator foi os híbridos superprecoces de milho (Flash e Penta), o segundo fator, espaçamentos entre linhas de milho $(0,45$ e $0,90 \mathrm{~m})$ e o terceiro fator, o controle de plantas daninhas em pós-emergência: 1. atrazine + foramsulfurom + iodosulfuron $(3000+45+3$ $\mathrm{g} \mathrm{ha}^{-1}$ i.a.); 2 . atrazine+ foramsulfurom + iodosulfuron $\left(2250+33,8+2,3\right.$ g ha ${ }^{-1}$ i.a. $) ; 3$. atrazine+ foramsulfurom+ iodosulfuron $\left(1500+22,5+1,5 \mathrm{~g} \mathrm{ha}^{-1}\right.$ i.a.); 4. testemunha capinada; 5. testemunha sem capina. Para as características morfológicas (estaturas e ângulo foliar vertical), o delineamento foi em blocos ao acaso com 40 repetições. As avaliações foram realizadas nas testemunhas capinadas, nos espaçamentos de 0,45 e $0,9 \mathrm{~m}$. 
A cultura do milho foi implantada em sucessão à cultura da aveia preta (Avena strigosa), a qual foi dessecada com uma aplicação de glyphosate $(720 \mathrm{~g}$ ha $^{-1}$ i.a.), realizada aos 12 dias antes da semeadura dos híbridos de milho. A semeadura e adubação na linha foram realizadas através de semeadora tracionada por trator. A adubação, no momento da semeadura, empregou 15, 68 e $64 \mathrm{~kg} \mathrm{ha}^{-1} \mathrm{de} \mathrm{N}, \mathrm{P}_{2} \mathrm{O}_{5}$ e $\mathrm{K}_{2} \mathrm{O}$. A adubação nitrogenada de cobertura foi realizada em duas parcelas de $60 \mathrm{~kg} \mathrm{ha}^{-1}$ de $\mathrm{N}$, quando havia quatro e oito folhas totalmente expandidas. Realizou-se o desbaste 30 dias após a semeadura, quando as plantas de milho estavam com cinco folhas totalmente expandidas, visando à obtenção de densidade de 65.000 plantas ha ${ }^{-1}$. As dimensões das parcelas utilizadas foram de $16,3 \mathrm{~m}^{2}$ para o espaçamento de $0,45 \mathrm{~m}$ e $27 \mathrm{~m}^{2}$ para o espaçamento de $0,90 \mathrm{~m}$, com adoção de sete linhas de semeadura por parcela, nos dois espaçamentos.

Os controles populacionais de percevejo barrigaverde (Dichelops spp) e lagarta-do-cartucho (Spodoptera frugiperda) foram obtidos com o inseticida metamidofós (900 $\mathrm{g} \mathrm{ha}^{-1}$ i.a.). A capina da testemunha e a aplicação dos herbicidas foram realizados quando os híbridos estavam com sete folhas completamente expandidas ( 25 dias após a emergência - DAE) A pulverização foi realizada com equipamento costal de precisão pressurizado com $\mathrm{CO}_{2}$, com vazão de $250 \mathrm{~L} \mathrm{ha}^{-1}$, aplicado com ponta de pulverização leque tipo 110.03 a uma pressão de $30 \mathrm{lb} \mathrm{pol}^{-2}$. No momento da aplicação, a temperatura média do ar foi igual a $27^{\circ} \mathrm{C}$ e a umidade relativa média do ar estava próxima a $70 \%$.

Estas avaliações das características morfológicas estatura e ângulo foliar vertical foram realizadas aos 38 e 72 DAE. A estatura foi determinada através de fita métrica, medindo da base do colmo até a base da última folha expandida. O ângulo foliar vertical foi medido entre a face adaxial da quinta folha expandida e o colmo da planta de milho, através de transferidor acoplado a hastes de madeira, elaborado especificamente para essa função.

A contagem total das plantas daninhas foi realizada em numa área de $0,16 \mathrm{~m}^{2}$ aos 13 e 34 dias após a aplicação dos herbicidas (DAA).

A matéria seca das plantas daninhas foi avaliada aos 50 DAA, coletando-se a parte aérea das espécies daninhas através de uma amostra de $0,16 \mathrm{~m}^{2}$ por parcela, para posterior secagem em estufa a $62{ }^{\circ} \mathrm{C}$ até obter-se massa constante. A matéria seca foi determinada através de balança com 0,001 g de precisão. $\mathrm{O}$ rendimento de grãos foi calculado pela massa de grãos obtida na área útil das parcelas, extrapolado para hectare, com posterior padronização da umidade para $13 \%$.
Os dados obtidos foram submetidos à análise de variância pelo teste $\mathrm{F}$ e, em caso de significância dos efeitos simples ou interações, realizou-se a análise complementar das médias dos tratamentos pelo teste de Tukey com $5 \%$ de probabilidade do erro experimental.

\section{RESULTADOS E DISCUSSÃO}

Houve diminuição na estatura das plantas de milho no espaçamento reduzido, em relação ao espaçamento convencional, aos 38 DAE. Entretanto, aos 58 DAE essa redução não foi verificada (Tabela 1). Não houve diferença entre os híbridos Penta e Flash quanto a esta característica. Também não houve diferenças significativas de ângulos foliares verticais entre os híbridos de milho (Tabela 1). No entanto, os ângulos foliares verticais foram menores (folhas mais eretófilas) no espaçamento de $0,9 \mathrm{~m}$ do que no de 0,45 $\mathrm{m}$, tanto para Penta quanto para Flash, nas duas avaliações efetuadas.

A principal espécie infestante da área experimental foi Brachiaria plantaginea (BRAPL), em densidades médias de 143 plantas $\mathrm{m}^{-2}$, em contagem efetuada na testemunha sem controle aos 13 DAA (Tabela 2), embora se tenha constado também infestações muito baixas de Euphorbia heterophylla.

Os híbridos utilizados não influenciaram o número de plantas de BRAPL por área, no entanto, houve interação entre espaçamento e níveis de controle de plantas daninhas, nas avaliações realizadas aos 13 e 34 dias após a aplicação (DAA) (Tabela 2). Na testemunha sem controle de plantas daninhas, aos 13 e $34 \mathrm{DAA}$, as densidades de BRAPL foram, respectivamente, $41 \%$ e $42 \%$ menores no espaçamento reduzido $(0,45 \mathrm{~m})$, em relação ao convencional $(0,9 \mathrm{~m})$. Nos demais níveis de controle de plantas daninhas, não houve diferenças de infestação de BRAPL entre os espaçamentos entre linhas, nem aos 13 DAA, nem aos 34 DAA.

A redução da infestação de BRAPL no espaçamento de $0,45 \mathrm{~m}$, na testemunha sem controle, foi devido, provavelmente, ao maior sombreamento nas entrelinhas da cultura do milho, visto que o fechamento mais rápido das entrelinhas reduz a emergência das plantas daninhas fotoblásticas positivas.

Com relação ao parâmetro matéria seca de plantas daninhas, avaliado aos 50 DAA (Tabela 3), houve interação tripla entre os fatores híbrido, espaçamento entre linhas e níveis de controle. O híbrido Penta foi mais eficiente do que Flash em reduzir a matéria seca de plantas daninhas no espaçamento de $0,9 \mathrm{~m}$, apenas no tratamento sem controle de plantas daninhas. 
Tabela 1. Características morfológicas e híbridos de milho em função do espaçamento entre linhas, aos 38 e 58 dias após a emergência (DAE)

\begin{tabular}{|c|c|c|c|c|}
\hline \multirow{3}{*}{ Híbridos } & \multicolumn{4}{|c|}{ Avaliações } \\
\hline & \multicolumn{2}{|c|}{$38 \mathrm{DAE}$} & \multicolumn{2}{|c|}{$58 \mathrm{DAE}$} \\
\hline & \multicolumn{2}{|c|}{ Espaçamento } & \multicolumn{2}{|c|}{ Espaçamento } \\
\hline & $0,45 \mathrm{~m}$ & $0,90 \mathrm{~m}$ & $0,45 \mathrm{~m}$ & $0,90 \mathrm{~m}$ \\
\hline & \multicolumn{4}{|c|}{ Estatura $(\mathrm{cm})$} \\
\hline Penta & $111 \mathrm{~b} \mathrm{~A}$ & 116 a A & 221 a A & 276 a A \\
\hline \multirow[t]{2}{*}{ Flash } & $111 \mathrm{~b} \mathrm{~A}$ & 145 a A & 224 a A & 224 a A \\
\hline & \multicolumn{4}{|c|}{ Ângulo foliar vertical $\left(^{\circ}\right)$} \\
\hline Penta & 45 a $\mathrm{A}$ & $41 \mathrm{~b} \mathrm{~A}$ & 45 a $\mathrm{A}$ & $41 \mathrm{~b} \mathrm{~A}$ \\
\hline Flash & 42 a $\mathrm{A}$ & $41 \mathrm{~b} \mathrm{~A}$ & 42 a A & $40 \mathrm{~b} \mathrm{~A}$ \\
\hline
\end{tabular}

Médias seguidas da mesma letra minúscula na linha, entre espaçamentos, e mesma letra maiúscula na coluna, entre híbridos, não diferem entre si pelo teste de Tukey a $5 \%$ de probabilidade do erro experimental, dentro de uma mesma época de avaliação.

Tabela 2. Densidade de Brachiaria plantaginea (BRAPL), em plantas $\mathrm{m}^{-2}$, em resposta a espaçamentos entre linhas e níveis de controle de plantas daninhas, aos 13 e 34 dias após a aplicação dos herbicidas (na média de dois híbridos de milho)

\begin{tabular}{|c|c|c|c|c|c|}
\hline \multirow{2}{*}{ Espaçamento } & \multicolumn{5}{|c|}{ Níveis de controle de plantas daninhas } \\
\hline & SC & $50 \%$ & $75 \%$ & $100 \%$ & $\mathrm{CC}$ \\
\hline & & & 13 DAA & & \\
\hline $0,45 \mathrm{~m}$ & 106 a B & 104 a $\mathrm{A}$ & $79 \mathrm{ab} \mathrm{A}$ & $80 \mathrm{ab} \mathrm{A}$ & $0 \mathrm{~b} \mathrm{~A}$ \\
\hline \multirow[t]{2}{*}{$0,90 \mathrm{~m}$} & 179 a $\mathrm{A}$ & $61 \mathrm{bc} \mathrm{A}$ & $96 \mathrm{~b} \mathrm{~A}$ & 72 bc A & 0 с A \\
\hline & & & 34 DAA & 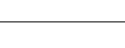 & \\
\hline $0,45 \mathrm{~m}$ & 49 a B & 49 a A & 62 a $\mathrm{A}$ & 71 a $\mathrm{A}$ & $0 \mathrm{~b} \mathrm{~A}$ \\
\hline $0,90 \mathrm{~m}$ & 84 a A & 49 a $\mathrm{A}$ & 64 a $\mathrm{A}$ & 46 a $\mathrm{A}$ & $0 \mathrm{~b} \mathrm{~A}$ \\
\hline
\end{tabular}

$\mathrm{SC}=$ Sem Capina; $50 \%=1500+22,5+1,5 \mathrm{~g} \mathrm{ha}^{-1}$ (atrazine + foramsulfuron + iodosulfuron); $75 \%=2250,0+33,8+2,3 ; 100 \%=3000,0+45,0$ $+3,0 ; \mathrm{CC}=$ Com Capina.

Médias seguidas da mesma letra minúscula na linha (níveis de controle) e mesma letra maiúscula na coluna (espaçamento) não diferem entre si pelo teste de Tukey a $5 \%$ de probabilidade. C.V. "níveis de controle" $=75 \%$ e C.V. "espaçamento" $=28 \%$ para avaliação realizada aos 13 DAA. C.V. "níveis de controle" = 32\% e C.V. "espaçamento" = 32\% para avaliação realizada aos 34 DAA.

Tabela 3. Matéria seca de plantas daninhas $\left(\mathrm{g} \mathrm{m}^{-2}\right)$ em resposta a híbridos, espaçamentos e níveis de controle, determinada aos 50 dias após a aplicação dos herbicidas

\begin{tabular}{|c|c|c|c|c|c|c|c|c|c|c|}
\hline \multirow{3}{*}{ Híbrido } & \multicolumn{10}{|c|}{ Espaçamento e níveis de controle } \\
\hline & \multicolumn{5}{|c|}{$0,45 \mathrm{~m}$} & \multicolumn{5}{|c|}{$0,90 \mathrm{~m}$} \\
\hline & SC & $50 \%$ & $75 \%$ & $100 \%$ & $\mathrm{CC}$ & SC & $50 \%$ & $75 \%$ & $100 \%$ & $\mathrm{CC}$ \\
\hline Penta & a247aA & b53aA & bc16aA & bc7aA & $\mathrm{c} 0 \mathrm{aA}$ & $\mathrm{a} 244 \mathrm{bA}$ & b32aA & b9aA & $\mathrm{b} 2 \mathrm{aA}$ & $\mathrm{b} 0 \mathrm{aA}$ \\
\hline Flash & $\mathrm{a} 150 \mathrm{aB}$ & ab58aA & bc $24 a A$ & bc16aA & $\mathrm{c} 0 \mathrm{aA}$ & $\mathrm{a} 483 \mathrm{aA}$ & $\mathrm{b} 62 \mathrm{aA}$ & bc21aA & c6aA & $\mathrm{cOaA}$ \\
\hline
\end{tabular}

$\mathrm{SC}=$ Sem Capina; $50 \%=1500+22,5+1,5 \mathrm{~g} \mathrm{ha}^{-1}$ (atrazine + foramsulfuron + iodosulfuron); $75 \%=2250,0+33,8+2,3 ; 100 \%=3000,0+45,0$ $+3,0 ; \mathrm{CC}=$ Com Capina.

Médias seguidas da mesma letra minúscula na coluna não diferem entre sí pelo teste de Tukey a 5\% de significância e comparam os híbridos dentro de um mesmo nível de controle e espaçamento. Médias seguidas da mesma letra maiúscula não diferem entre sí pelo teste de Tukey a 5\% de significância e comparam os espaçamentos dentro do mesmo nível de controle e híbrido. Médias antecedidas da mesma letra minúscula na linha não diferem entre sí pelo teste de Tukey a $5 \%$ de significância e comparam os níveis de controle dentro do mesmo espaçamento e híbrido. 
Na testemunha sem capina do híbrido Flash, o espaçamento de $0,45 \mathrm{~m}$ foi $31 \%$ mais eficiente em reduzir a matéria seca de plantas daninhas do que no de $0,9 \mathrm{~m}$, efeito não observado no híbrido Penta (Tabela 3). Esse fato demonstra que a utilização de espaçamentos reduzidos entre linhas, com a finalidade de se obter maior supressão de plantas daninhas é mais apropriada para o híbrido Flash, enquanto para Penta, a supressão de plantas daninhas não é afetada pela variação nos espaçamentos entre linhas.

Os dados de matéria seca de plantas daninhas revelam que a redução no seu desenvolvimento pela diminuição do espaçamento entre linhas é dependente do híbrido. Comparando os mesmos espaçamentos em várias combinações de herbicidas aplicados em pósemergência precoce e/ou tardia, no entanto, utilizando os híbridos Penta e Sprint, verificou-se que, em Sprint, a matéria seca de plantas daninhas no espaçamento de 0,45 e $0,9 \mathrm{~m}$ foi, respectivamente, de 1,6 e $54,8 \mathrm{~g} \mathrm{~m}^{-2}$, nos tratamentos em pós-emergência precoce de atrazine+simazine. No entanto, não foi constatada diferença entre os espaçamentos para o híbrido Penta (Trezzi et al., 2008). Para o híbrido Pioneer 3751IR, a redução de espaçamento de 76 para $51 \mathrm{~cm}$ não reduziu a infestação nem o crescimento das plantas daninhas, assim como não influenciou no momento de aplicacão dos herbicidas (JoHnSOn e HOVERSTAD, 2002)

Como a superioridade de Penta, em relação a Flash, em suprimir a matéria seca de plantas daninhas ocorreu no espaçamento de $0,9 \mathrm{~m}$, e não em $0,45 \mathrm{~m}$, como seria esperado, é provável que outras características de Penta, não determinadas no presente experimento, como a largura, o comprimento e a disposição azimutal das folhas, tenham contribuído para as diferenças entre os híbridos. Assim, a primeira hipótese desse trabalho, de que características como o ângulo foliar, que influenciam a interceptação de radiação solar, poderiam modificar a infestação de plantas daninhas na cultura, não foi comprovada.

A maior dose da associação de atrazine + foramsulfuron + iodosulfuron não resultou em redução significativa da população de BRAPL (Tabela 2) nem da matéria seca total de plantas daninhas (Tabela 3), em relação às demais doses, com exceção da matéria seca no espaçamento de $0,9 \mathrm{~m}$ e híbrido Flash, em que houve diferença entre o nível mais baixo e o mais alto da associação de herbicidas. Preliminarmente, verificase no experimento a possibilidade da redução de $25 \%$ da dose de herbicidas em todas as combinações de híbridos e espaçamentos; no entanto, a redução de doses não esteve vinculada à redução do espaçamento entre linhas. Assim, a segunda hipótese, de que a menor infestação de plantas daninhas nos espaçamentos mais estreitos permitiria a redução de doses de herbicidas não foi comprovada. Em experimento utilizando os híbridos Penta e Sprint, a redução do espaçamento entre linhas de $0,90 \mathrm{~m}$ para $0,45 \mathrm{~m}$ também não esteve associada à possibilidade de redução de doses dos herbicidas atrazine+simazine (TREZZI et al., 2008).

Em relação ao rendimento de grãos, a análise da variância foi significativa para efeito simples de níveis de controle e para a interação entre híbrido e espaçamento entre linhas. O rendimento de grãos do tratamento sem capina (SC) foi $18 \%$ menor do que a média dos demais tratamentos (Tabela 4). Não houve diferenças de rendimento de grãos entre os níveis de atrazine+foramsulfuron+iodosulfuron, e nem destes em relação ao tratamento capinado (Tabela 4).

Tabela 4. Rendimento de grãos em função dos níveis de controle de plantas daninhas (na média de dois dos híbridos e dois espaçamentos)

\begin{tabular}{lc}
\hline Níveis de controle & Produtividade \\
\hline & $\mathrm{kg} \mathrm{ha}^{-1}$ \\
$50 \%$ & $8493 \mathrm{~b}$ \\
$75 \%$ & $9878 \mathrm{a}$ \\
$100 \%$ & $10079 \mathrm{a}$ \\
$\mathrm{CC}$ & $10528 \mathrm{a}$ \\
\hline
\end{tabular}

$\mathrm{SC}=$ Sem capina; $50 \%=1500+22,5+1,5 \mathrm{~g} \mathrm{ha}^{-1}$ (atrazine + foramsulfuron + iodosulfuron); $75 \%=2250,0+33,8+2,3 ; 100 \%=$ $3000,0+45,0+3,0 ;$ CC $=$ Com capina.

Médias com a mesma letra não diferem entre si pelo teste de Tukey a $5 \%$ de probabilidade.

Tanto para Penta quanto para Flash, a variação no espaçamento entre linhas de 0,45 para $0,90 \mathrm{~m}$ não provocou mudanças no rendimento de grãos (Tabela 5). O híbrido Penta foi $17 \%$ mais produtivo do que Flash no espaçamento de $0,9 \mathrm{~m}$, no entanto, não houve diferenças entre os híbridos no espaçamento de $0,45 \mathrm{~m}$ (Tabela 5). Esse resultado pode estar relacionado à vantagem competitiva sobre plantas daninhas de Penta, em relação a Flash, no espaçamento convencional (Tabela 3 ).

Tabela 5. Rendimento de grãos $\left(\mathrm{kg} \mathrm{ha}^{-1}\right)$ em função de híbridos e espaçamentos (médias dos níveis de controle de plantas daninhas)

\begin{tabular}{lcc}
\hline \multirow{2}{*}{ Híbrido } & \multicolumn{2}{c}{ Espaçamento entre linhas } \\
\cline { 2 - 3 } & 0,45 & 0,90 \\
\cline { 2 - 3 } Penta & 9821 a A & 10774 a A \\
Flash & 10043 a A & 9225 a B \\
\hline
\end{tabular}

Médias seguidas da mesma letra minúscula na linha (espaçamento) e mesma letra maiúscula na coluna (híbrido) não diferem entre si pelo teste de Tukey a $5 \%$ de probabilidade. 
Os resultados de experimentos testando a redução do espaçamento entre linhas sobre o rendimento de grãos de milho são bastante heterogêneos. Em alguns estudos constatam-se aumentos expressivos de rendimento de grãos com a redução do espaçamento entre linhas (SHRESTHA et al., 2001; Johnson e Hoverstad, 2002; Strieder, 2006), enquanto em outros não há esse incremento (TEASDALE, 1995; Trezzi et al., 2008). Fatores como o híbrido e a população de plantas (ARGENTA et al., 2001a) e a época de semeadura (SANGOI et al., 2001) podem contribuir para a variabilidade de resultados.

\section{CONCLUSÕES}

1. O ângulo foliar de plantas de milho tornase mais planófilo com a adoção de espaçamentos entre linhas reduzidos, mas outras características de plantas de milho estão envolvidas no desempenho dos espaçamentos.

2. O uso do espaçamento de $0,45 \mathrm{~m}$ entre linhas não contribui para a redução dos níveis de herbicidas para os híbridos Flash e Penta, mas sua adoção beneficia o manejo de plantas daninhas, especialmente para híbridos com arquitetura mais ereta como o híbrido Flash.

\section{REFERÊNCIAS}

ALMEIDA, M.L.; MERROTO JR., A.;SANGOI, L.; ENDER, M.; GUIDOLIN, A.F. Incremento na densidade de plantas: uma alternativa para aumentar o rendimento de grãos de milho em regiões de curta estação estival de crescimento. Ciência Rural, v.30, p.23-29, 2000.

ARGENTA, G.; SILVA, P.R.F.; BORTOLINI, C.G.; FORSTHOFER, E.L.; MANJABOSCO, E.A.; NETO, V.B. Resposta de híbridos simples de milho à redução do espaçamento entre linhas. Pesquisa Agropecuária Brasileira, v.36, p.71-78, 2001a.

ARGENTA, G.; SILVA, P.R.F.; SANGOI, L. Arranjo de plantas em milho: análise do estado da arte. Ciência Rural, v.31, p.10751084, 2001b.

BALLARÉ, C.L.; SCOPEL A.L.; SANCHEZ R.A. Far-red radiation reflected from adjacent leaves: an early signal of competition in plant caopies. Science, v.247, p.329331, 1990.

BEGNA, S.H.; HAMILTON, R.I.; DWYER, L.M.; STEWART, D.W.; CLOUTIER, D.; ASSEMAT, L.; FOROUTAN-POUR, K.; SMITH, D.L. Weed biomass production response to plant spacing and corn (Zea mays) hybrids differing in canopy architecture. Weed Technology, v.15, p.647-653, 2001.

JOHNSON, G.A.; HOVERSTAD, T.R. Effect of row spacing and herbicide application timing on weed control and grain yield in corn (Zea mays). Weed Technology, v.16, p.548-553, 2002.
KUNZ, J.H.; GERGONCI, J.I.; BERGAMASCHI, H.; DALMAGO, G.A.; HECKLER, B.M.M.; COMIRAN, F. Uso da radiação solar pelo milho sob diferentes preparos do solo, espaçamento e disponibilidade hídrica. Pesquisa Agropecuária Brasileira, v.42, p.1511-1520, 2007.

MADDONNI, G.A.; OTEGUI, M.E.; CIRILO, A.G. Plant population density, row spacing and hybrid effects on maize canopy architecture and light attenuation. Field Crops Research, v.71, p.183-193, 2001.

MADDONNI, G.A.; OTEGUI, M.E.; ANDRIEU, B.; CHELLE, M.; CASAL, J.J. Maize leaves turn away from neighbors. Plant Physiology, v.130, p.1181-1189, 2002.

RAJCAN, I.; CHANDLER, K.J.; SWANTON, C.J. Red-far-red ratio of reflected light: a hypothesis of why early-season weed control is important in corn. Weed Science, v.52, p.774778, 2004.

SANGOI, L.; ENDER, M.; GUIDOLIN, A.F.; ALMEIDA, M.L.; HEBERLE, P.C. Influence of row spacing reduction on maize grain yield in regions with a short summer. Pesquisa Agropecuária Brasileira, v.36, p.861-869, 2001.

SANGOI, L.; SILVA, P.R.F.; ARGENTA, G.; HORN, D. Bases morfo-fisiológicas para aumentar a tolerância de cultivares de milho a altas densidades de plantas. In: REUNIÃO TÉCNICA CATARINENSE DE MILHO E FEIJÃO, 4, 2003, Lages, SC. Resumos expandidos... Lages, SC: CAV-UDESC, 2003. p.19-24.

SHRESTHA, A.; RAJCAN, I.; CHANDLER, K.; SWANTON, C.J. An integrated weed management strategy for glufosinate-resistant corn (Zea mays). Weed Technology, v.15, p.517-522, 2001.

SMITH, H. Phytochromes and light signal perception by plants: an emerging synthesis. Nature, v.407, p.585591, 2000.

STRIEDER, M.L. Resposta do milho à redução do espaçamento entre linhas em diferentes sistemas de manejo. 2006. 94 f. Dissertação de mestrado (Mestrado em Fitotecnia) - Programa de Pós-Graduação em Fitotecnia, Faculdade de Agronomia, Universidade Federal do Rio Grande do Sul, Porto Alegre, 2006.

TEASDALE, J.R. Influence of narrow row/high population corn on weed control and light transmittance. Weed Technology, v.9, p.113-118, 1995.

TREZZI, M.M.; VIDAL, R.A.; NUNES,A.L.; PRATES, M.V.B; GUSTMAN, M.R., ARGENTA, G. Manejo químico de plantas daninhas na cultura do milho em função de características morfofisiológicas e redução de espaçamento da cultura. Planta Daninha, v.26, n.4, p.845-853, 2008.

VIDAL, R.A. Ação dos herbicidas. Volume 1: Absorção, translocação e metabolização. Porto Alegre: Ribas Vidal, 2002. 89 p. 